\title{
Diacronie
}

Studi di Storia Contemporanea

$\mathrm{N}^{\circ} 20,4 \mid 2014$

II diritto militante

\section{Introduzione n. 20 - dicembre 2014}

\section{Maria Malatesta}

\section{(2) OpenEdition}

\section{Journals}

\section{Edizione digitale}

URL: http://journals.openedition.org/diacronie/1668

DOI: 10.4000/diacronie.1668

ISSN: 2038-0925

\section{Editore}

Association culturelle Diacronie

\section{Notizia bibliografica digitale}

Maria Malatesta, «Introduzione n. 20 - dicembre 2014 », Diacronie [Online], № 20, 4 | 2014, Messo online il 01 décembre 2014, consultato il 23 avril 2019. URL : http://journals.openedition.org/ diacronie/1668; DOI : 10.4000/diacronie.1668

Questo documento è stato generato automaticamente il 23 aprile 2019.

Creative Commons License 


\section{Introduzione n. 20 - dicembre 2014}

Maria Malatesta

IL DIRITTO MILITANTE AVVOCATI E MAGISTRATI NEL XX E XXI SECOLO 
1 Il filo rosso che lega i saggi contenuti in questo dossier è il diritto militante. Il corso di Storia delle istituzioni politiche e sociali della Laurea magistrale in Scienze storiche tenuta nell'anno accademico 2013-2014 da Francesca Sofia ha affrontato questa tematica all'interno del più vasto tema del processo politico partendo dalle suggestioni contenute nel volume seminale di Liora Israel (Le armi del diritto, Milano, Giuffrè 2012) e percorrendo nuove piste di ricerca. I saggi, i cui autori sono tutti studenti di quel corso, hanno messo in luce aspetti poco conosciuti o trascurati del diritto militante fornendo nel complesso un quadro storiografico non privo di caratteri innovativi.

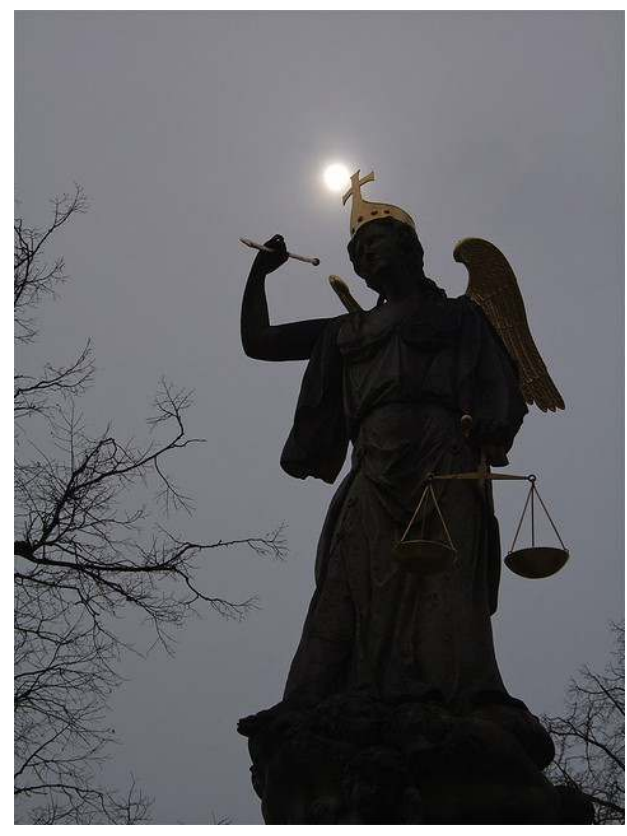

2 È stata avvertita innanzi tutto l'esigenza di dotare il tema di coordinate teoriche in grado di fornire una migliore comprensione di alcuni casi storici presi in esame. A svolgere questo compito è William Mazzaferro, che nel suo saggio fa una lucida ricostruzione della concezione del diritto nella teoria marxista. Nonostante le difficoltà dei padri fondatori del marxismo a rapportarsi con il diritto borghese e a mettere a punto un uso proletario del diritto, nella prima metà del Novecento furono i partiti comunisti europei a detenere un monopolio quasi totale della difesa militante e a usare i processi come arene in cui potevano essere valutate molteplici strategie politiche.

3 Si inserisce all'interno di questa problematica la sezione di saggi scritti da Gianluigi Briguglio, Nicola Caroli, Simeone Del Prete e Greta Fedele e incentrati sulla figura dell'avvocato militante italiano per antonomasia, il bolognese Leonida Casali. Grazie ad uno scavo approfondito attuato nell'archivio dell'avvocato (depositato presso l'Istituto Parri di Bologna), il saggio ricostruisce le dinamiche che legarono Casali al Partito comunista nella sua attività di difensore degli ex-partigiani nei processi in cui erano accusati di atti di violenza compiuti alla fine della seconda guerra mondiale soprattutto in Emilia Romagna. Casali riuscì a mantenere un'autonomia professionale di fronte al PCI soprattutto grazie alla posizione che si era guadagnato all'interno del partito; nonostante ciò, il suo profilo di avvocato militante fu condizionato in modo decisivo dalla militanza politica e dal rapporto con il partito.

4 Grazie ai saggi di Francesco Mantovani, Marina De Ghantuz Cubbe e Costanza Zanasi è possibile ricostruire, seppure a grandi linee, il percorso durante il quale il PCI perse il monopolio della difesa militante e prese piede dai primissimi anni Settanta un nuovo concetto di diritto militante, non più coincidente con la difesa dei comunisti colpiti dalla repressione delle classi dominanti, ma aperto a nuove realtà e soggetti sociali. Nel corso degli anni Settanta la salute, il lavoro, l'ambiente sono emersi come grandi scenari nei quali avvocati e magistrati si sono incontrati in un comune progetto incentrato sulla difesa di nuovi diritti. Il saggio di Federico Mantovani ricostruisce l'impegno di Magistratura democratica profuso negli anni Settanta nella rivista «Quale giustizia» per il riconoscimento dei diritti dei malati psichiatrici e l'abbattimento dell'istituzione 
manicomiale. De Ghantuz Cubbe e Zanasi analizzano dal canto loro due processi che hanno segnato la storia del nostro paese, quello del Petrolchimico di Porto Marghera e quello sull'Eternit. Il saggio di De Ghantuz Cubbe ha il pregio di mettere per la prima volta a fuoco in una prospettiva storiografica il ruolo svolto dai periti e l'uso della scienza fatto da costoro in questa tipologia di processi i cui esiti dipendono dalla decisione dei magistrati di optare per l'una o l'altra delle perizie di parte sui danni commessi o meno da determinate sostanze sulla salute dei lavoratori e dei cittadini. La ricostruzione del lungo iter processuale sulla fabbrica piemontese dell'amianto compiuta da Costanza Zanasi fa emergere il profilo di Sergio Bonetto, il prototipo dell'avvocato militante del XXI secolo, che ha dedicato la sua vita professionale alla difesa dei lavoratori e dei cittadini vittime delle stragi compiute dal capitalismo.

Pochi giorni fa la Corte di Cassazione ha nei fatti annullato la sentenza d'appello del processo Eternit, nella quale era stato riconosciuto il danno che la fabbrica aveva arrecato alla salute dei lavoratori e dei cittadini. La notizia ha scosso profondamente l'opinione pubblica e ha mostrato con assoluta evidenza che oggi come ieri, il diritto militante che lotta in difesa dei deboli, degli oppressi, degli esclusi è un terreno nel quale si combattono battaglie all'ultimo sangue.

\section{AUTORE}

\section{MARIA MALATESTA}

Laureata in Filosofia nel 1972 presso l'Università di Bologna. Professore ordinario di Storia contemporanea nella Facoltà di Lettere e Filosofia. Materie di insegnamento: Storia contemporanea, Storia delle istituzioni sociali, Storia delle professioni. Ex coordinatore del Dottorato in Storia e geografia d'Europa, coordina dal XXVI ciclo il Dottorato in storia. Titolare dell'insegnamento di Storia contemporanea nell'a.a. 1995-1996 presso l'Université Denis DiderotParis 7. Professore invitato nel 2000 presso l'Ecole Normale Superiéure; nel 2001 e nel 2003 presso la Maison des Sciences de l'Homme; professore invitato all'Ecole des Hautes Etudes en Sciences Sociales nel 2008. E'stato membro della Commissione per la storia dell'avvocatura del Consiglio Nazionale Forense dal 199 al 2007; è attualmernte membro del Consiglio Italiano delle Scienze sociali e del comitato scientifico dell'Istituto per la storia di Bologna; Coordinatore scientifico del Centro di ricerca sulla storia delle professioni (http://www.ceprof.unibo.it/). Ha partecipato come responsabile di un'unità di ricerca locale a due progetti ex-40\% (1991-1994, 1998-1999) e come membro locale al progetto PRIN 2005-2006 su Migrazioni maschili, migrazioni femminili: per una cartografia della mobilità geografica in Italia tra età moderna e contemporanea. Coordinatore nazioanale del progetto PRIN 2007 "Le professioni:dal progetto della politica alla politica del quotidiano". Coordinatore del progetto strategico finanziato nel 2007 dall'ateneo di Bologna dal titolo Atlante storico delle professioni. Ha partecipato dal 1987 al 1991 al gruppo di ricerca sulla Storia sociale europea diretto da Hartmut Kaelble (Freie Universität Berlin). Ha partecipa dal 2004 al 2018 al gruppo di ricerca diretto da Yves Dezalay (CNRS- Maison des Sciences de l'Homme- Paris) e Brian Garth (American Bar Foundation) sulle trasformazioni dei campi giuridici internazionali. Collabora dal 2007 con il Centre de sociologie Maurice Halbwachs 
- CNRS- Paris -Ecole des Hautes Etudes en sciences sociales. Nel 1996 ha insegnato per un semestre Storia contemporanea all'Università di Paris VII- Denis Diderot- Paris. Dal 2000 al 2011 è' stata più volte professeur invitée all' Ecole Normale Superieure e all'Ecole des Hautes Etudes en sciences sociales. Membro del comitato scientifico delle seguenti riviste "Actes de la Recherche en Sciences Sociales”, “Comparative Sociology", "Le Mouvement Social", "Società e Storia". 